\title{
ECOGRAFIA TRANSFONTANELAR COM FLUXO A CORES EM RECÉM-NASCIDOS PREMATUROS
}

\author{
Marcelo Cardoso de Assis', Helio Rubens Machado²
}

\begin{abstract}
RESUMO - Com o objetivo de determinarmos, evolutivamente, a medida da velocidade do fluxo sangüíneo nas artérias intracranianas, em recém-nascidos prematuros (RNP) normais e com hemorragia intracerebral, avaliamos - no período de junho de 1994 a março de 1999 - 73 recém-nascidos prematuros. A idade gestacional variou de 28 a 36 semanas e o peso ao nascimento variou de $720 \mathrm{~g}$ a $2530 \mathrm{~g}$. 0 diagnóstico da hemorragia intracerebral foi realizado utilizando-se a ecografia transfontanelar (EGT). Para avaliação seqüencial da medida da velocidade do fluxo sangüíneo nas artérias intracranianas os 73 foram submetidos a EGT, com Doppler pulsátil, no $3^{\circ}, 7^{\circ}, 30^{\circ}$ e $90^{\circ}$ dias de vida. Após obtermos os valores numéricos destas velocidades determinamos o indice de resistência (IR). Ao analisarmos os valores do IR, comparando-se os 2 grupos de RNP, concluimos que os valores do IR são sempre mais elevados nos RNP normais (RNP-N) que nos RNP com hemorragia intracerebral (RNP-HIC); que tanto no grupo de RNP normais quanto no grupo de RNP com hemorragia intracerebral os valores do IR decrescem significativamente com o acréscimo da idade dos neonatos. Analisando-se ainda, comparativamente, os valores do IR nos RNP com hemorragia intracerebral, em seus diversos graus, observamos não haver, evolutivamente, diferença estatisticamente significante. Analisando-se também, comparativamente, os valores do IR nos RNP com hemorragia intracerebral localizada no hemisfério cerebral direito ou esquerdo concluimos não haver diferença estatisticamente significante entre os valores do IR obtidos das artérias localizadas no hemisfério cerebral acometido comparados aos valores do IR obtidos do hemisfério cerebral não afetado.
\end{abstract}

PALAVRAS-CHAVE: recém-nascidos prematuros, hemorragia intracerebral, ecografia transfontanelar, Doppler pulsátil.

\section{Intracranial blood flow velocities evaluated by color doppler (duplex) in preterm infants}

ABSTRACT - In order to ascertain the blood flow velocities in the intracranial arteries we evaluated 73 preterm neonates during a period ranging from June 1994 to March 1999. These preterm infants were divided in two separate groups, 18 healthy and 55 with intracranial hemorrhage. They were subjected to sequencial measurements of blood flow velocities in the intracranial arteries. The gestational age of the whole group varied from 28 to 36 weeks and birth weights between 720 and $2530 \mathrm{~g}$. The diagnosis of the intracerebral hemorrhages in these preterm neonates were done using high resolution gray and color scale transfontanellar ultrasonography brain scans. The ultrasound evaluations were performed in the initial 3rd, 7th and 14th day of life. The 73 preterm infants were evaluated with sequencial measurements of blood flow velocity in the intracranial arteries using the Doppler technique through the anterior fontanelle. Doppler evaluation of the cerebral vessels were performed on days 3, 7, 30 and 90 of life. These evaluations were performed in the six intracranial arteries, meaning: right and left anterior and middle cerebral arteries and right and left internal carotid arteries. Doppler recordings were made using Duplex Color-Doppler system, pulse echo probe of 3,5; 5,0 and 7,5 MHz. Measuring the blood flow velocity in the cerebral arteries we obtained a maximum systolic velocity and end diastolic velocity with a rate in meters per second $(\mathrm{m} / \mathrm{s})$ for each cardiac cycle. After obtaining these numerical values for these velocities we obtained the resistance index (RI) or Pourcelot index. In a progressive way as the resistance index (RI) values were being obtained in each stage of this study they were also being checked in the cerebral arteries of healthy preterm infants and infants with intracranial hemorrhages. We also analyzed in a comparative method the values of the resistive index between the two groups of preterm infants observing their behaviour.The results obtained when comparing the RI values in the various arteries during the different stages of the study permitted us to conclude that the RI values of healthy pre-term infants were always larger than the RI values of pre-term infants with intracranial hemorrhage. We also conclude that the RI values in the healthy pre-term infants and in the preterm infants with intracranial hemorrhages decreased progressively with the increasing age of neonates.

KEY WORDS: preterm infants, intracranial hemorrhage, transfontanellar ultrasonography, duplex color-Doppler.

\footnotetext{
1Professor Adjunto, Doutor da Disciplina de Neurologia da Faculdade de Medicina da Universidade Federal de Uberlândia, Uberlândia MG, Brasil; ${ }^{2}$ Professor Associado da Disciplina de Neurocirurgia, Departamento de Anatomia e Cirurgia da Faculdade de Medicina de Ribeirão Preto da Universidade de São Paulo, Ribeirão Preto SP, Brasil.
}

Recebido 24 Março 2003, recebido na forma final 27 Agosto 2003. Aceito 27 Setembro 2003.

Dr. Marcelo Cardoso de Assis - Disciplina de Neurologia - Faculdade de Medicina da Universidade Federal de Uberlândia - Bloco 2 H, Campus Umuarama - 38701-136 Uberlândia MG - Brasil. 
A hemorragia intracerebral (HIC) é importante causa de mortalidade e morbidade em recém-nascidos prematuros (RNP) particularmente naqueles com peso inferior a $1500 \mathrm{~g}$ e idade gestacional abaixo de 32 semanas. Sua incidência, neste grupo de risco, atinge percentuais que variam de 31 a $65 \%$. Associa-se a uma mortalidade de 1 a 2,5 por 1000 nascidos vivos e sua morbidade relaciona-se com a ocorrência de hidrocefalia, paralisia cerebral e retardo mental. Considera-se como hemorragia intracerebral aquelas hemorragias subependimárias (da matriz germinal), intraventriculares e intraparenquimatosas. A primeira classificação das hemorragias intraventriculares e subependimárias em RNP foi proposta por Papile et al. ${ }^{1}$ que as classificou em 4 graus: Grau I - hemorragia subependimária; Grau II - hemorragia intraventricular, sem dilatação dos ventrículos, Grau III - hemorragia intraventricular, com dilatação dos ventrículos; Grau IV hemorragia intraventricular, com hemorragia intraparenquimatosa.

Para o diagnóstico das enfermidades neurológicas no período neonatal podemos utilizar a Ecografia Transfontanelar (EGT), a Tomografia Computadorizada (TC) e a Ressonância Magnética Nuclear (RMN). Para o estudo do fluxo sangüíneo, a utilização da técnica de Ultra-Som Doppler (US-Doppler) iniciou-se com os estudo de Satomura ${ }^{2}$ e posteriormente Franklin et al. ${ }^{3}$, que descreveram sua utilização em angiologia e neurologia. Peronneau et al. ${ }^{4}$ foram os introdutores das aparelhagens de US-Doppler pulsátil possibilitando tecnicamente o estudo da velocidade do fluxo sangüíneo. Para o estudo da resistência vascular periférica, utilizando-se a técnica de US-Doppler, Pourcelot ${ }^{5}$ introduziu o conceito de índice de resistência (IR), que tornou-se matematicamente expresso por: $I R=(S-D)$ / $S$, onde $S=$ velocidade sistólica máxima e $D=$ velocidade diastólica final. A primeira descrição da utilização da técnica de EGT-Doppler para avaliação do fluxo pulsátil em uma artéria intracraniana deve-se a Bada et al. ${ }^{6}$. Estes autores estudaram a fisiopatologia da hemorragia intracerebral e intraventricular em neonatos, sob condições clínicas de hipoxemia, partindo do princípio de que modificações na velocidade do fluxo, nas artérias localizadas intracranialmente, seriam indicativas de alterações na circulação cerebral .

Este estudo teve como objetivos: determinar, pela técnica de Dopplervelocimetria, os valores do IR nas artérias cerebrais anteriores direitas e esquerdas, cerebrais médias direitas e esquerdas e carótidas internas direitas e esquerdas, em RNP normais e com hemorragia intracerebral. Avaliar evolutivamente os valores do IR em RNP normais nos 3 primeiros meses de vida. Avaliar evolutivamente os valores do IR em RNP com hemorragia intracerebral nos 3 primeiros meses de vida. Comparar os valores do IR, ao longo dos 3 primeiros meses de vida, entre o grupo de RNP normais e com hemorragia intracerebral, observando sua evolução. Comparar os valores do IR, nos grupos de RNP com hemorragia intracerebral grau I, grau II, grau III e grau IV, observando sua evolução. Comparar os valores do IR nos grupos de RNP com hemorragia intracerebral, localizada distintamente no hemisfério cerebral direito ou esquerdo, observando sua evolução.

\section{MÉTODO}

Foram examinados e avaliados evolutivamente, através da técnica de EGT-Color-Doppler (EGT-CD) 100 recémnascidos prematuros, no período compreendido entre junho de 1994 a março de 1999. A idade gestacional variou de 28 a 36 semanas e o peso ao nascimento de 720 $\mathrm{g}$ a $2530 \mathrm{~g}$.

Estes RNP foram divididos em 2 grupos:

$I^{\circ}$ Grupo - Composto por 45 RNP normais, avaliados por EGT-CD nos 3 primeiros dias e entre o sétimo e décimo quarto dia de vida extra-uterina, nos quais obteve-se o índice de resistência nas artérias intracranianas.

Sub-grupo la - Composto por 18 RNP normais, pertencentes ao Grupo I, que foram acompanhados até o $3^{\circ}$ mês de vida extra-uterina.

${ }{ }^{\circ}$ Grupo - Composto por 55 RNP com diagnóstico ecográfico de hemorragia intracerebral, classificadas em grau I, II, III ou IV (segundo Papile') que foram acompanhadas até o $3^{\circ}$ mês de idade, para avaliação seqüencial do índice de resistência, obtido através da medida da velocidade do fluxo sangüíneo nas artérias intracranianas.

Todos os 100 RNP incluídos em nosso estudo submeteram-se à avaliação neonatológica, com anotação dos seguintes dados: idade gestacional, peso ao nascimento, sexo, índice de Apgar no $1^{\circ}$ e $5^{\circ}$ minutos, perímetro craniano e tipo de parto. Foram excluídos do estudo todos os RNP portadores de qualquer enfermidade que sabidamente pudesse interferir com os valores da medida da velocidade do fluxo sangüíneo nas artérias intracranianas, tais como, persistência do ducto arterioso, policitemia, síndrome da angústia respiratória e pneumotorax.

Para avaliação ecográfica utilizamos aparelhagem de US bidimensional com Doppler pulsátil colorido (EGTColor-Doppler), transdutor de 3,5 a 10,0 MHz, modelo Siemens Sonoline VERSA PRO (Siemens Medical Systems, Inc. Ultrasound Group).

Todos os 100 RNP foram avaliados, pela primeira 
vez, dentro dos 3 primeiros dias de vida extra-uterina com estudo ecográfico craniano para diagnóstico das hemorragias intracerebrais. Posteriormente foram avaliados no $7^{\circ}$ e $14^{\circ}$ dias de vida quando então foram divididos nos 2 grupos já citados.

O Grupo I foi formado por 45 recém-nascidos prematuros sem hemorragia intracerebral e foi avaliado por EGT-CD no $1^{\circ}$ dia e/ou no $2^{\circ}$ dia e no $3^{\circ}$ dia de vida extrauterina e, posteriormente, entre o $7^{\circ}$ e o $14^{\circ}$ dia de vida com a finalidade de excluir-se o diagnóstico de HIC. As velocidades dos fluxos sangüíneos nas artérias cerebrais anteriores direitas (ACAD) e esquerdas (ACAE), cerebrais médias direitas (ACMD) e esquerdas (ACME) e carótidas internas direitas (ACID) e esquerdas (ACIE) foram determinadas, obrigatoriamente, no $3^{\circ}$ dia de vida. Utilizamos estas medidas para determinarmos, como parâmetro próprio, os valores do índice de resistência em RNP normais. Este grupo foi subdividido em um subgrupo la, compostos por 18 entre os 45 RNP normais, sendo avaliados pela técnica de EGT-CD no $3^{\circ}$ dia de vida extra-uterina e sucessivamente no $7^{\circ}, 30^{\circ}$ e $90^{\circ}$ dias de vida extra-uterina, para analisarmos o comportamento do índice de resistência nesta faixa etária, em RNP normais .

O Grupo II foi composto pelos 55 RNP que apresentaram HIC e também foram avaliados no $3^{\circ}$ dia de vida extra-uterina e, posteriormente, no $7^{\circ} \mathrm{dia}, 30^{\circ}$ dia e $90^{\circ}$ dias de vida extra-uterina, com a mesma finalidade.

Técnica de ecografia transfontanelar-color-doppler - Todos os estudos com EGT-Color-Doppler (Dopplervelocimetria) foram realizados através da fontanela anterior, utilizada como janela acústica, com estudo vascular em cortes coronais e sagitais. Os estudos e registros da velocidade do fluxo sangüíneo foram realizados nas seguintes artérias: artéria cerebral anterior em seu segmento distal representado pela artéria pericalosa, bilateralmente, artéria cerebral média em seus segmentos M1 e segmentos distais bilateralmente e artérias carótidas internas direita e esquerda.

Análise estatística - $\mathrm{Na}$ análise estatística utilizamos o teste t pareado e comparamos os valores obtidos do IR em cada artéria avaliada, em cada etapa do estudo, entre os grupos de RNP normais e com hemorragia intracerebral. Utilizamos o mesmo teste para compararmos os valores obtidos do IR em cada artéria avaliada, em cada etapa do estudo, entre os grupos de RNP com hemorragia intracerebral em seus vários graus. Utilizamos também a análise de variância com medidas repetidas para avaliar, evolutivamente, o comportamento dos valores do IR obtidos em cada uma das artérias, dentro do mesmo grupo de RN (prematuros normais e prematuros com hemorragia intracerebral), bem como entre o grupo de RNP com hemorragia intracerebral localizada no hemisfério cerebral direito ou esquerdo. As comparações foram consideradas estatisticamente significantes com valores de $p<0.05$.

\section{RESULTADOS}

A determinação do IR no grupo de 45 RNP normais com idade gestacional variando de 28 a 37 semanas, realizada no $3^{\circ}$ dia de vida extra-uterina mostrou-nos, como média, valores de $0,83 \pm 0,07$ para a artéria cerebral anterior direita; $0,85 \pm 0,07$ para a artéria cerebral anterior esquerda; $0,83 \pm 0,07$ para a artéria cerebral média direita; $0,84 \pm 0,07$ para a artéria cerebral média esquerda; $0,86 \pm 0,06$ para a artéria carótida interna direita e valores de 0,85 $\pm 0,06$ para a artéria carótida interna esquerda.

No grupo de RNP normais quando comparamos os valores do IR obtidos nas diversas fases do estudo, para as diversas artérias avaliadas, obtivemos os seguintes resultados:

1) Artéria cerebral anterior direita: decréscimo do IR com diferença estatisticamente significante $(p<0.05)$ quando comparados os valores obtidos no $3^{\circ} \mathrm{com}$ o $90^{\circ} \mathrm{dia}, \mathrm{no} 7^{\circ} \mathrm{com}$ o $90^{\circ}$ dia e no $30^{\circ} \mathrm{com}$ o $90^{\circ}$ dia de vida. Ao comparararmos os valores obtidos no $3^{\circ} \mathrm{com}$ o $7^{\circ}$ dia e no $7^{\circ} \mathrm{com}$ o $30^{\circ}$ dia de vida não há diferença estatisticamente significante.

2) Artéria cerebral anterior esquerda: decréscimo do IR com diferença estatisticamente significante $(p<0.05)$ quando comparados os valores obtidos no $3^{\circ} \mathrm{com}$ o $90^{\circ}$ dia de vida e no $7^{\circ} \mathrm{com}$ o $90^{\circ}$ dia de vida.

3) Artéria cerebral média direita: decréscimo do IR com diferença estatisticamente significante $(p<$ $0.05)$ quando comparados os valores obtidos no $3^{\circ}$ com o $30^{\circ} \mathrm{dia}, 3^{\circ} \mathrm{com}$ o $90^{\circ} \mathrm{dia}$, no $7^{\circ} \mathrm{com}$ o $30^{\circ} \mathrm{dia}$, no $7^{\circ} \mathrm{com}$ o $90^{\circ}$ dia e no $30^{\circ} \mathrm{com}$ o $90^{\circ}$ dia de vida.

4) Artéria cerebral média esquerda: decréscimo do IR com diferença estatisticamente significante $(p<0.05)$ quando comparados os valores obtidos no $3^{\circ} \mathrm{com} 30^{\circ} \mathrm{dia}$, no $3^{\circ} \mathrm{com}$ o $90^{\circ}$ dia e no $7^{\circ} \mathrm{com}$ o $90^{\circ}$ dia de vida.

5) Artéria carótida interna direita: decréscimo do IR com diferença estatisticamente significante $(p<0.05)$ quando comparados os valores obtidos no $3^{\circ} \mathrm{com} 30^{\circ} \mathrm{dia}, 3^{\circ} \mathrm{com} 90^{\circ} \mathrm{dia}, 7^{\circ} \mathrm{com} 30^{\circ}$ dia e $7^{\circ}$ com $90^{\circ}$ dia de vida.

6) Artéria carótida interna esquerda: decréscimo do IR com diferença estatisticamente significante $(p<0.05)$ quando comparados os valores obtidos no $3^{\circ} \mathrm{com}$ o $30^{\circ} \mathrm{dia}, 3^{\circ} \mathrm{com}$ o $90^{\circ} \mathrm{dia}, 7^{\circ} \mathrm{com}$ o $30^{\circ}$ dia, $7^{\circ}$ com o $90^{\circ}$ dia e $30^{\circ}$ com o $90^{\circ}$ dia de vida.

No grupo de RNP com hemorragia intracerebral quando comparamos os valores do IR obtidos nas diversas fases do estudo, para as diversas artérias avaliadas, obtivemos os seguintes resultados: 
1) Artéria cerebral anterior direita: decréscimo do IR com diferença estatisticamente significante $(p<0.05)$ quando comparados os valores obtidos no $3^{\circ}$ com o $90^{\circ}$ dia de vida.

2) Artéria cerebral anterior esquerda: decréscimo do IR com diferenças estatisticamente significantes $(p<0.05)$ quando comparados os valores obtidos no $3^{\circ} \mathrm{com}$ o $30^{\circ} \mathrm{dia}, 3^{\circ} \mathrm{com}$ o $90^{\circ} \mathrm{dia}, 7^{\circ} \mathrm{com}$ $90^{\circ}$ dia e $30^{\circ} \mathrm{com} 90^{\circ}$ dia de vida.

3) Artéria cerebral média direita: decréscimo do IR com diferenças estatisticamente significantes $(p<0.05)$ quando comparados os valores obtidos no $3^{\circ} \mathrm{com}$ o $7^{\circ} \mathrm{dia}, 3^{\circ} \mathrm{com}$ o $30^{\circ} \mathrm{dia}, 3^{\circ} \mathrm{com}$ o $90^{\circ} \mathrm{dia}$, $7^{\circ} \mathrm{com}$ o $90^{\circ}$ dia e $30^{\circ} \mathrm{com}$ o $90^{\circ}$ dia de vida.

4) Artéria cerebral média esquerda: decréscimo do IR com diferenças estatisticamente significantes $(p<0.05)$ quando comparados os valores obtidos no $3^{\circ} \mathrm{com} \circ 7^{\circ} \mathrm{dia}, 3^{\circ} \mathrm{com}$ o $90^{\circ} \mathrm{dia}, 7^{\circ} \mathrm{com}$ o $90^{\circ} \mathrm{dia}$ e $30^{\circ} \mathrm{com}$ o $90^{\circ}$ dia de vida.

5) Artérias carótidas internas direitas e esquerdas: apresentaram comportamento semelhante, com decréscimo do IR com diferenças estatisticamente significantes $(p<0.05)$ quando comparados os valores obtidos no $3^{\circ} \mathrm{com}$ o $7^{\circ} \mathrm{dia}, 3^{\circ} \mathrm{com}$ o $30^{\circ} \mathrm{dia}, 3^{\circ} \mathrm{com}$ o $90^{\circ} \mathrm{dia}, 7^{\circ} \mathrm{com} \circ 90^{\circ}$ dia e $30^{\circ} \mathrm{com}$ o $90^{\circ}$ dia de vida. Nestas duas artérias só não houve diferença estatisticamente significante quando comparados os valores do IR obtidos em avaliações realizadas no $7^{\circ}$ dia com aqueles obtidos no $30^{\circ}$ dia de vida.

Subdividindo o grupo de RNP com hemorragia intracerebral de acordo com o grau da hemorragia (grau I, II, III e IV, segundo Papile') e analisando evolutivamente o IR nas diversas artérias intracranianas avaliadas observamos não existir diferença estatisticamente significante entre os valores do IR, independentemente do grau da hemorragia .

Ao analisarmos o grupo de RNP com hemorragia intracerebral dividindo-o em 2 subgrupos, de acordo com a localização da hemorragia - se no hemisfério cerebral direito ou esquerdo - não observamos diferença estatisticamente significante entre os valores do IR obtidos das artérias localizadas no hemisfério cerebral acometido pela hemorragia comparando-os com os valores obtidos no hemisfério não afetado, nas várias artérias intracranianas avaliadas, ao longo do estudo, independentemente do grau da hemorragia.

\section{DISCUSSÃO}

A utilização da ecografia transfontanelar para diagnóstico das hemorragias intracerebrais em neo- natos encontra-se já consolidada na prática neurológica, pediátrica e, sobretudo, em neonatologia, devido à sua sensibilidade e especificidade em detectar esta enfermidade e também por possibilitar o seu acompanhamento evolutivo. Reveste-se ainda de outras características de benignidade ressaltando-se ser um método propedêutico que evita expor o RN à radiação e a procedimentos anestésicos/sedativos. A possibilidade de estudo da medida da velocidade do fluxo sangüíneo nas artérias cerebrais pela técnica de dopplervelocimetria, acoplada ao exame de ultra-sonografia em tempo real, expandiu em muito a aplicabilidade deste recurso propedêutico e possibilitou inúmeros estudos sobre as modificações da velocidade do fluxo sangüíneo nas artérias intracranianas, tanto em RNP normais como em RNP com hemorragia intracerebral.

Em nosso estudo avaliamos evolutivamente o IR em um grupo de 18 RNP normais, ao longo dos 3 primeiros meses de vida, nas seis artérias intracranianas. Ao compararmos os valores obtidos das avaliações realizadas no $3^{\circ}$ e no $7^{\circ}$ dia de vida não observamos diferença estatisticamente significante. Observamos, quando comparamos os valores do IR obtidos das artérias cerebrais médias e carótidas internas que, a partir do $7^{\circ}$ dia até o $90^{\circ}$ dia de vi$\mathrm{da}$, ocorreu um decréscimo progressivo e estatisticamente significante nos valores do IR. Para as artérias cerebrais anteriores os valores do IR decresceram com diferenças estatisticamente significantes a partir da idade de 30 dias.

Bada et al. ${ }^{6}$ estudando evolutivamente o IR em um grupo de RNP normais, ao longo dos 7 primeiros dias de vida, com determinações seqüenciais do IR no $1^{\circ}, 2^{\circ}, 3^{\circ}$ e $7^{\circ}$ dias, observaram que não existem variações significativas dos valores do IR nesta faixa etária. Van Bel et al. ${ }^{7}$ realizando estudos prospectivos em um grupo de RNP normais determinaram evolutivamente o IR na artéria cerebral anterior ao longo dos 5 primeiros dias de vida. Observaram estes pesquisadores que o IR pode decrescer nas primeiras 32 horas de vida para depois estabilizarse ao longo dos 5 dias de vida. Evans et al. ${ }^{8}$ estudaram a velocidade do fluxo sangüíneo e o índice de resistência em RN a termo com baixo peso ao nascimento, nas artérias cerebrais anteriores e médias, ao longo da primeira semana de vida. Observaram estes autores que a média dos valores do IR foram similares nas artérias avaliadas e que o IR decresce progressivamente na primeira semana de vida, marcadamente entre o $1^{\circ}$ e o $3^{\circ}$ dias. Ley et al. ${ }^{9}$ realizaram estudos onde avaliaram evolu- 
tivamente a velocidade do fluxo sangüíneo nas artérias cerebrais anteriores e médias, durante os 3 primeiros dias de vida, em $21 \mathrm{RN}$ a termo sendo que 8 foram classificados como pequenos para a idade gestacional e 13 como apropriados para a idade gestacional. As avaliações foram realizadas com 1 hora, 4, 12, 24, 48 e 72 horas após o nascimento. Ao comparar os valores do IR, em ambos os grupos de neonatos, foram observados que os valores do IR decresciam progressivamente até 72 horas de idade, em todas as artérias avaliadas.

Analisamos, ainda, no grupo de RNP normais, se existiria alguma diferença estatisticamente significante entre os valores do IR, nas várias artérias avaliadas, distintamente entre as artérias do hemisfério cerebral direito comparadas às artérias do hemisfério cerebral esquerdo. Concluímos não existir, em qualquer estágio do estudo, diferenças estatisticamente significantes entre os valores do IR neste grupo de RNP. Winberg et al. ${ }^{10}$ estudaram a velocidade do fluxo sangüíneo nas artérias cerebrais, utilizando a técnica de EGT-Doppler, em dois grupos de RN normais, a termo e prematuros. Obtiveram as avaliações utilizando o sistema doppler de fluxo pulsátil e de ondas contínuas e observaram que, independentemente da técnica utilizada, os valores obtidos para a velocidade do fluxo sangüíneo nas artérias carótidas internas intracranianas eram equivalentes. Além deste parâmetro procuraram avaliar se modificações no posicionamento do crânio poderiam interferir nos resultados obtidos. Concluíram não haver nenhuma modificação na velocidade do fluxo sangüíneo cerebral relacionada à rotação do crânio e, tampouco, se a velocidade do fluxo era obtidas nas artérias localizadas à direita ou à esquerda.

A avaliação evolutiva do IR no grupo de 55 RNP-HIC, ao longo dos 3 primeiros meses de vida, mostrou-nos que para as artérias cerebrais médias e carótidas internas os valores do IR decrescem significativamente comparando-se desde o $3^{\circ} \mathrm{com}$ o $7^{\circ}$ dia de vida e assim sucessivamente, até o $90^{\circ}$ dia de vida. Os valores do IR obtidos das artérias cerebrais anteriores decresceram, com diferenças estatisticamente significantes, a partir da idade de 30 dias. Bada et al. ${ }^{6}$ relataram que em um grupo de RNP-HIC por eles estudados os valores do IR foram elevados por volta do $3^{\circ}$ dia de vida (época de suas hemorragias) e que retornaram aos seus valores normais por volta do $7^{\circ}$ dia de vida. Ando et al. ${ }^{11}$ estudaram as modificações pós-natais da velocidade do fluxo sangüíneo cerebral em recém- nascidos prematuros normais e com diversas enfermidades. Ao determinarem o IR na artéria cerebral anterior, em RNP com hemorragia subependimária, estes autores observaram que seu valor era baixo durante as 6 primeiras horas de vida e permaneciam estáveis posteriormente. Para o grupo de RNP com hemorragia intraventricular estes autores observaram que o IR era elevado durante as primeiras 6 horas de vida pós-parto e, então, decrescia progressivamente.

Ao analisarmos o grupo de RNP com hemorragia intracerebral, subdividindo-o de acordo com o grau da hemorragia, observamos não existir nenhuma diferença estatisticamente significante entre os valores do IR, analisados distintamente para cada artéria intracraniana avaliada, em cada etapa do estudo. Assim é que os valores do IR, obtidos ao longo dos 3 primeiros meses de idade, naqueles RNP com hemorragia grau I quando comparados com os valores do IR daqueles RNP com hemorragia grau II, ou III, ou grau IV, não apresentaram nenhuma diferença estatisticamente significante, independentemente da artéria ou da fase do estudo considerada. Mires et al. ${ }^{12}$ realizaram estudos em um grupo de 57 RNP que foram avaliados durante os 10 primeiros dias de vida pela técnica de EGTdoppler, com determinação do IR nas artérias cerebrais anteriores e cerebrais médias. A primeira avaliação ultra-sonográfica foi realizada com 1 hora de vida e as seguintes com 12, 24, 48 e 72 horas de vida e, posteriormente, no $6^{\circ}, 7^{\circ}$ e $10^{\circ}$ dias de vida. Neste grupo de RN 18 apresentaram hemorragia periventricular. Ao comparar os resultados do IR obtidos nas artérias cerebrais anteriores e médias de 14 RNP com hemorragia grau I/II, com um grupo de RNP normais, estes autores não encontraram nenhuma diferença estatisticamente significante. Para o grupo de RNP com hemorragia grau III e IV (04 pacientes) os valores do IR eram significativamente elevados, quando comparados com o grupo de RNP normais.

Ao analisarmos comparativamente os valores do IR obtidos nas seis artérias intracranianas, no grupo de 55 RNP com hemorragia intracerebral, separando-os de acordo com a localização da hemorragia (se no hemisfério cerebral direito ou esquerdo ) não observamos nenhuma diferença estatisticamente significante, independentemente dos valores analisados terem sido obtidos das artérias localizados ou não no hemisfério cerebral acometido.

Kuban et al..$^{13}$ estudando um grupo de RNP, ao longo dos 3 primeiros dias de vida, determinaram 
o IR na artéria cerebral anterior e também um coeficiente de variação utilizado de 5 a 20 ondas pulsáteis obtidas ao doppler. Este coeficiente de variação foi utilizado como uma medida do grau de flutuação na velocidade do fluxo sangüíneo cerebral. Ao compararem os valores dos coeficientes de variação para as artérias do hemisfério cerebral direito e do esquerdo, em grupos distintos de RN, (sem hemorragia cerebral, com hemorragia intracerebral unilateral e com hemorragia intracerebral bilateral), concluíram que o coeficiente de variação do lado da hemorragia não diferia significativamente daquele do lado não afetado.

Ao compararmos os dois grupos de RNP, normais e com hemorragia intracerebral, observamos que ocorreram diferenças estatisticamente significantes entre os valores do IR obtidos nas avaliações realizadas no $7^{\circ}$ dia, sempre com valores do IR mais elevados no grupo de RNP normais. Teria alguma relevância maior o fato de que, nas avaliações realizadas no $3^{\circ}$ dia de vida, os RNP dos dois grupos apresentem valores do IR sem diferença estatisticamente significante e, considerando-se a avaliação seqüencial, já no $7^{\circ}$ dia de vida os valores do IR serem significativamente menores no grupo de RNP com HIC? Dos trabalhos de Bada et al. ${ }^{6}$ e de Pourcelot et al. ${ }^{14}$ sabemos que o IR pode ser considerado como uma medida relativa da velocidade do fluxo sangüíneo, devendo ser indicativo da resistência vascular. E que valores elevados do IR correlacionam-se com vasoconstricção e baixa velocidade do fluxo sangüíneo, enquanto que baixos valores do IR correlacionam-se com vasodilatação e alta velocidade do fluxo sangüíneo. Estes valores significativamente menores do IR, obtidos no $7^{\circ}$ dia de vida, nas artérias cerebrais do grupo de RNP que apresentavam hemorragia intracerebral, poderia explicar a evolução com acentuação destas hemorragias em quatro de nossos pacientes. Perlman et al. ${ }^{15}$ estudaram RNP com manifestações clínicas da síndrome da angústia respiratória com o intuito de observar se modificações na velocidade do fluxo sangüíneo cerebral ocorreriam na vigência desta enfermidade e, em ocorrendo, se estariam relacionadas ao surgimento de hemorragia intraventricular. Concluíram estes autores que existe uma correlação entre a presença de flutuações na velocidade do fluxo sangüíneo cerebral e o subsequente desenvolvimento de hemorragia intraventricular. Estudos experimentais realizados por Goddard-Finegold et al. ${ }^{16}$ com realização de hipotensão induzida demonstraram também a ocorrência de hemorragia periventricular, levando à suposição de que as reduções no fluxo sangüíneo cerebral em RNP podem resultar em infarto da matriz germinal e que a reperfusão desta área acarretaria hemorragias. Archer et al. ${ }^{17}$ avaliaram, pela técnica de EGTdoppler, um grupo de $43 \mathrm{RN}$ a termo com manifestações clínicas de hipoxia perinatal. Estes RN foram avaliados desde as primeiras horas de vida com acompanhamento subsequente e os objetivos deste estudo foram de correlacionar se possíveis alterações no IR, determinado nas primeiras 48 horas de vida, poderiam predizer alterações neurológicas tardias neste grupo de pacientes. Concluíram que naqueles $\mathrm{RN}$ com hipoxemia, comparados com RN normais, a técnica de US-doppler foi capaz de predizer as seqüelas neurológicas tardias, com um acerto de $86 \%$. Estes autores relataram que após hipoxia perinatal alguns RN apresentaram baixos valores do IR, e que todos os valores anormais do IR foram observados antes de 72 horas de vida. Van Bel et al. ${ }^{7}$ ao analisarem a importância das alterações do fluxo sangüíneo cerebral, no desenvolvimento e extensão das hemorragias peri e intraventriculares, concluíram que existe uma estreita relação entre as flutuações na velocidade do fluxo sangüíneo cerebral, a subsequente diminuição do IR e a progressão das hemorragias peri e intraventriculares. Estes mesmos autores, estudando um grupo de 23 RNP com hemorragia intracerebral, através do estudo evolutivo da velocidade do fluxo sangüíneo cerebral observaram que, comparandoos com um grupo de 32 RNP normais, os valores do IR eram significativamente mais baixos nos prematuros com hemorragia intracerebral, em avaliações realizadas com 32, 40 e 60 horas de vida.

Todos estes dados permitem-nos considerar que a determinação seriada do índice de resistência, obtido a partir do estudo evolutivo da medida da velocidade do fluxo sangüíneo nas artérias intracranianas, utilizando-se a técnica não invasiva de EGT-CD, fornecem-nos informações seguras acerca das modificações na circulação cerebral em recémnascidos prematuros. E que, ao permitir a observação das modificações e flutuações na velocidade do fluxo sangüíneo cerebral, em recém-nascidos com determinadas enfermidades que predispõem à ocorrência da hemorragia intracerebral - tais como a síndrome da angústia respiratória, aumentos na pressão venosa central e na pressão arterial média - possibilite ao neonatologista instituir medidas terapêuticas preventivas que reduzam a sua ocorrência ou minorem a sua gravidade. 


\section{REFERÊNCIAS}

1. Papile LA, Burstein J, Burstein R, Koffler H. Incidence and evolution of subependymal and intraventricular hemorrhage: a study of infants with birth weights less than $1500 \mathrm{gm}$. J Pediatr 1978;92:529-534.

2. Satomura S. Study on the flow patterns in peripheral arteries by ultrasonics. J Acoust Soc JPN 1959;15:151-158.

3. Franklin DL, Schlegal WA, Rushmer RF. Blood flow measured by Doppler frequency shift of backscattered ultrasound. Science 1961;134:564-565.

4. Peronneau P. Debitmetrie ultrasonore : Développements et application expérimentales. Europ Surg Res 1969;1:147-156.

5. Pourcelot L. Diagnostic ultrasound for cerebral vascular diseases. In Donaldi J and Levis S (eds). Present and future of diagnostic ultrasound. Rotterdam: Kooyter, 1976:141-147.

6. Bada HS, et al. Noninvasive diagnosis of neonatal asphyxia and intraventricular hemorrage by Doppler ultrasound. Pediatrics 1979;95:775779 .

7. Van Bel F, Van de Bor M, Stijnen T, Baan J, Ruys JH. Aetiological role of cerebral blood-flow alterations in development and extension of peri-intraventricular haemorrhage. Develop Med Child Neurol 1987:29:601-614.

8. Evans DH, Levene MI, Shortland DB, Archer LNJ. Resistance index, blood flow velocity, and resistance area product in the cerebral arteries of very low birth weight infants during the first week of life. Ultrasound Med Biol 1988;14:102-110.
9. Ley D, Marsal K. Doppler velocimetry in cerebral vessels of small for gestational age infants. Early Hum Dev 1992;31:171-180.

10. Winberg P, Dahlstrom A, Lundell B. Reproducibility of intracranial Doppler flow velocimetry. Acta Paediatr Scand 1986;329 (Suppl):S134S139.

11. Ando Y, Takashima S, Takeshita K. Cerebral blood flow velocity in preterm neonates. Brain Dev 1985;7:385-391.

12. Mires GJ, Patel NB, Forsyth JS, Howie PW. Neonatal cerebral Doppler flow velocity waveforms in the pre-term infant with cerebral pathology. Early Hum Dev 1994;36:213-222.

13. Kuban KCK, Skouteli H, Cherer A, et al. Hemorrhage, phenobarbital, and fluctualting cerebral blood flow velocity in the neonate. Pediatrics 1988;82:548-553.

14. Pourcelot L, Santini JJ, Saliba E, Billard C, Gold F, Laugier J. Mesure non invasive du flux sanguin cérébral chez le nouveau-né hydrocéphale. Neurochirurgie 1985;31:07-13.

15. Perlman JM, Goodman S, Kreusser KL, Volpe JJ. Reduction in intraventricular hemorrhage by elimination of fluctuating cerebral blood-flow velocity in preterm infants with respiratory distress syndrome. N Eng J Med 1985;312:1353-1357.

16. Goddard-Finegold J, Michael LH. Cerebral blood flow and experimental intraventricular haemorrhage. Pediatr Res 1984;18:7-11

17. Archer LNJ, Levene MI, Evans DH. Cerebral artery Doppler ultrasonography for prediction of outcome after perinatal asphyxia. Lancet 1986;15:1116-1117 